\title{
Avaliação clínica do uso de solução salina hipertônica no tratamento da acidose láctica ruminal aguda em bovinos
}

\author{
Clinical evaluation of hypertonic saline solution treatment for acute ruminal lactic acidosis \\ on cattle
}

\begin{abstract}
Frederico Augusto Mazzocca Lopes RODRIGUES ${ }^{1}$; Antonio Humberto Hamad MINERVINO ${ }^{1}$; Raimundo Alves BARRÊTO JÚNIOR ${ }^{2}$; Alexandre Coutinho ANTONELLI ${ }^{3}$; Leonardo Frasson dos REIS ${ }^{1}$; Carolina Akiko Sato Cabral ARAUUJ' ${ }^{1}$; Rodrigo Nogueira Fernades FERREIRA ${ }^{1}$; Thales dos Anjos Faria VECHIATO ${ }^{1}$; Clara Satisuki MORI $^{1}$; Enrico Lippi ORTOLANI ${ }^{1}$
\end{abstract}

\author{
${ }^{1}$ Departamento de Clínica Médica, Faculdade de Medicina Veterinária e Zootecnia da \\ Universidade de São Paulo, São Paulo - SP, Brasil \\ ${ }^{2}$ Universidades Federal Rural do Semi-Árido, Mossoró - RN, Brasil \\ ${ }^{3}$ Universidade Federal do Vale do São Francisco, Petrolina-PE, Brasil
}

\begin{abstract}
Resumo
A infusão de solução salina hipertônica (SSH) é um importante tratamento em animais com choque hipovolêmico. Bovinos com acidose láctica ruminal aguda (ALRA) frequentemente apresentam quadros de desidratação. Este trabalho avaliou a eficiência do tratamento da ALRA com SSH. Foram empregados 12 bovinos machos, mestiços com um ano de idade. Após implantação de cânula ruminal e período de adaptação alimentar os animais foram submetidos à indução de ALRA por meio de administração de sacarose no rúmen. Após 20 horas da indução, os animais foram aleatoriamente divididos em dois grupos. Um deles (SSH) foi tratado na $20^{\text {a }} \mathrm{h}$ com $5 \mathrm{~mL} / \mathrm{kg} \mathrm{P.V}$. de uma solução de $\mathrm{SSH}(7,5 \% \mathrm{NaCl})$, dentro de $15 \mathrm{~min}$, e em seguida $20 \mathrm{~mL} / \mathrm{kg} / \mathrm{P}$.V. de solução salina isotônica (SSI), no decorrer dos próximos 165 minutos. O outro grupo (SSI) foi medicado da mesma forma, com exceção da SSH que foi substituído por $5 \mathrm{~mL} / \mathrm{kg} \mathrm{PV}$ de SSI. Cinco litros de conteúdo ruminal foram retirados, sendo repostos com cinco litros de água em ambos os grupos. Variáveis foram mensuradas no momento 0 (MO), na $20^{\mathrm{a}} \mathrm{h}$ (M20h) e no decorrer dos tratamentos (M30', M60', M120' e M180'). O uso de SSH provocou, nos primeiros 30 minutos, uma ligeira acidemia, acompanhada de discreta hipercapnia, contudo sem provocar efeitos colaterais. A infusão de SSH provocou uma queda no volume globular, confirmando a migração de fluidos do rúmen para a circulação, corrigindo parcialmente a desidratação. Assim, o uso de SSH é uma possibilidade de tratamento adicional da desidratação provocada pela ALRA.
\end{abstract}

Palavras-chave: Hipertônica. Desidratação. Acidose. Láctica. Ruminal. Bovinos.

\begin{abstract}
Hypertonic saline solution (HSS) is known as an important treatment for hypovolemic shock. Cattle with acute rumen lactic acidosis (ARLA) usually present different degrees of dehydration. This study evaluated the efficiency of HSS for treatment of ARLA. Twelve yearling, cross-bred, male cattle were used. After an adaptation period, when a rumen cannula was implanted, the animals were submitted to an induction of ARLA by the administration of sucrose into the rumen. Twenty hours later the cattle were randomly divided in two groups. The $1^{\text {st }}$ group was treated with $5 \mathrm{~mL} / \mathrm{kg}$ BW with $7.5 \%(\mathrm{NaCl})$, within 15 min after $20^{\text {th }} \mathrm{h}$ of the induction; thereafter it was infused $20 \mathrm{~mL} / \mathrm{kg} \mathrm{BW}$ of isotonic saline solution (ISS) for the next $165 \mathrm{~min}$. The $2^{\text {nd }}$ group was treated only with ISS. Five liters of ruminal contents were removed and replaced with five liters of water in both groups. Variables were measured at time 0 (MO), $20 \mathrm{~h}$ (M20h) and throughout the treatment (M30', M60', M120' e M180'). The use of HSS caused a mild acidemia followed by a discrete hypercapnia. No side effects were seen in cattle treated with HSS. Infusion of HSS decreased the globular volume indicating a passage of fluids from the rumen into the bloodstream, correcting partially the dehydration. The use of HHS is a possible additional treatment for correction of dehydration caused by ARLA.
\end{abstract}

Keywords: Hypertonic. Dehydration. Acidosis. Lactic. Ruminal. Cattle.

Correspondência para:

Prof. Dr. Enrico Lippi Ortolani

Departamento de Clínica Médica

Faculdade de Medicina Veterinária e Zootecnia

Universidade de São Paulo. USP

Rua Prof. Dr. Orlando Marques de Paiva, 87, São Paulo - SP
CEP 05508270

e-mail.ortolani@usp.br

Recebido: $16 / 06 / 2010$

Aprovado: 09/11/2011 


\section{Introdução}

O tratamento da acidose láctica ruminal aguda consiste na correção da acidose metabólica sistêmica e da desidratação. A acidemia é corrigida com o uso de soluções tampões, tais como: bicarbonato de sódio e lactato $\mathrm{L}(+)^{1}$.

Devido à migração de água que se instala nos ruminantes com acidose ocasionado um quadro de desidratação, o sucesso do tratamento depende entre outros fatores da correção da desidratação que se instala nos animais ${ }^{2}$. Os autores que revisaram o assunto indicaram a expansão e reposição dos fluidos como fundamental, apontando que a hidratação parenteral seja feita com soluções isotônicas ${ }^{3}$.

O uso de solução salina hipertônica (SSH) tem sido advogado para o tratamento de vários tipos de choque (hemorrágico, endotóxico e hipovolêmico) e em quadros de desidratação em várias espécies animais inclusive no homem, desde o trabalho pioneiro de um grupo de pesquisa brasileiro ${ }^{4}$.

Basicamente, esta solução hipertônica contendo 7,2 $\%$ de cloreto de sódio $(2.400 \mathrm{mOsm} / \mathrm{L})$ tem como função, rapidamente repor o volume plasmático por meio do deslocamento de água livre do espaço intracelular e trato gastrintestinal para o leito vascular. Esse tratamento traz vários benefícios hemodinâmicos, entre eles: uma rápida expansão do volume plasmático, vasodilatação temporária, melhora na pressão arterial, estímulos respiratórios e cardíacos e aumento na taxa de filtração glomerular ${ }^{5}$ Desde este estudo, muitos autores avaliaram os efeitos da solução hipertônica e sua superioridade em comparação a outros líquidos para reposição volêmica ${ }^{5,6,7,8}$.

Apesar da grande quantidade de estudos utilizando a SSH tenham sido realizados em monogástricos e bezerros lactentes, poucos, até o momento, tiveram como foco de pesquisa os ruminantes adultos ${ }^{5,6,8}$. Para esta última categoria tem sido indicado o uso de $\mathrm{SSH}$ para na correção de estados severos de desidratação, em especial em consequência às diarreias, choque hemorrágico e endotóxico ${ }^{5}$.

Embora o tratamento com SSH possa induzir uma suave acidose metabólica sanguínea, pois geralmente o pH deste fluido diminui menos de 0,08 unidades de $\mathrm{pH}$, esta situação é temporária e rapidamente se dissipa, sendo por isso considerada de pequena importância clínica ${ }^{5}$.

Huber ${ }^{9}$ verificou em ovinos com ALRA um aumento de osmolaridade do conteúdo ruminal, passando de $255 \mathrm{mOsm} / \mathrm{L}$, em condições normais, para $402 \mathrm{mOsm} / \mathrm{L}$ durante o pico da ALRA. A osmolaridade do rúmen passou a ser maior (em média 109 $\mathrm{mOsm} / \mathrm{L}$ ) que a encontrada no sangue (em torno de $297 \mathrm{mOsm} / \mathrm{L}$ ). Essa diferença de osmolaridade entre os compartimentos provocou uma enorme migração de fluidos internos do corpo do animal para o rúmen ocasionando desidratação no animal.

Mendes Netto e Ortolani ${ }^{2}$ compararam o tratamento da ALRA com solução de bicarbonato de sódio ou solução comercial de ringer com lactato. Enquanto que a última solução se apresentava $272 \mathrm{mOsm} / \mathrm{L}$, a de bicarbonato continha $573 \mathrm{mOsm} / \mathrm{L}$, ou seja, ligeiramente hipertônica em relação ao sangue. Nesse trabalho ficou evidente que, na $2^{\text {a }}$ hora após o início do tratamento, a solução de bicarbonato provocou significativa diminuição nos valores de volume globular em relação aos animais tratados com solução de ringer com lactato. Essa queda no volume globular foi interpretada como melhora na correção do estado de desidratação, devido a um ligeiro aumento na osmolaridade sanguínea, deslocando água do espaço intersticial para o espaço intravascular.

Até o momento nenhum estudo foi conduzido para avaliar o papel da SSH na correção do estado de desidratação desenvolvida durante a ALRA. Espera-se que esse tratamento tenha um efeito benéfico. Tal hipótese é oriunda dos resultados do experimento conduzido por Roeder, Su e Schaalje ${ }^{8}$, em dez vacas que foram submetidas a jejum hídrico por 36 horas e, 
em seguida, tratadas com água diretamente no rúmen ou água neste órgão mais SSH parenteral. Os autores concluíram que existiu uma evidência nítida que a SSH provocou uma maior absorção de água ruminal, pela diminuição dos valores de volume globular e aumento significativo no volume circulatório, na perfusão renal, filtração glomerular e volume urinário nos bovinos tratados com SSH.

\section{Material e Método}

Foram utilizados 12 bovinos, machos, inteiros, mestiços, hígidos, com cerca de um ano de idade e pesando aproximadamente $250 \mathrm{~kg}$. Dois meses antes do início do período experimental, todos os animais receberam aplicação de endectocida a base de ivermectina e vacina contra clostridiose.

Os ruminantes foram submetidos à cirurgia para colocação de cânula ruminal de látex. Após os procedimentos cirúrgicos, os garrotes passaram por um período de adaptação às instalações e ao novo manejo alimentar. Durante tal período e no decorrer do período experimental, os animais foram alimentados com uma ração total composta de $65 \%$ da matéria seca (MS) de feno de capim Coast-cross e 35\% da MS de ração concentrada comercial com $14 \%$ de proteína bruta. A quantidade de matéria seca da ração total ofertada aos animais foi calculada em relação a 2,7 \% do peso vivo individual, sendo corrigido no decorrer do ensaio. Os animais receberam esta dieta por, no mínimo, quatro meses antes do início da indução fornecida duas vezes ao dia. O estudo clínico foi realizado no Galpão de Doenças Nutricionais e Metabólicas da FMVZ-USP, campus de São Paulo, Estado de São Paulo. Os animais foram mantidos em sistema de tie-stall.

O delineamento experimental foi inteiramente casualizado, com distribuição dos animais em dois grupos, o controle com solução salina isotônica (n =6) e o tratado com solução salina hipertônica $(\mathrm{n}=$ 6). O modelo de indução do quadro de acidose láctica ruminal seguiu o protocolo de administração de sacarose no rúmen ${ }^{10} \mathrm{~A}$ fim de minimizar o risco de morte dos animais a quantidade de sacarose foi diminuída em $15 \%$.

Vinte horas após a indução com sacarose foi o momento considerado como estabelecimento do quadro de acidose láctica, iniciando-se então o tratamento médico dos animais. Foram retirados cinco litros de conteúdo ruminal e acrescentado o mesmo volume de água segundo protocolo de Roeder, Su e Schaalje ${ }^{8}$. Em seguida os animais foram tratados com infusão, pela cânula intravascular, de $5 \mathrm{~mL} / \mathrm{kg}$ de P.V. (peso vivo) de solução salina hipertônica no grupo SSH e mesmo volume de solução salina isotônica $(\mathrm{NaCl} 0,9 \%)$ para o grupo controle SSI, no decorrer de 15 minutos, seguido de $20 \mathrm{~mL} / \mathrm{kg}$ de P.V. de solução salina isotônica no decorrer das três horas de acompanhamento em ambos os grupos. Para permitir a infusão total do volume de SSH ou SSI nos 15 minutos iniciais foi utilizada bolsa pressurizadora (marca $\left.\mathrm{VBM}^{\circledR}\right)$. Após este período, para corrigir a acidose sistêmica, os animais foram medicados com solução isotônica de bicarbonato de sódio $\left(\mathrm{NaHCO}_{3} 1,3 \%\right.$ ), com base no resultado do exame hemogasométrico.

Foi realizado exame clínico e colheitas de sangue e conteúdo ruminal no decorrer do ensaio, nos seguintes momentos: Basal (M0), imediatamente antes da indução de quadro de ALRA; momento 20 horas (M20h), 20 horas após a indução de ALRA, no momento crítico da ALRA; momento 30 minutos (M30'), 30 minutos após o início do tratamento; momento 60 minutos (M60'), 60 minutos após o início do tratamento; momento 120 minutos (M120'), 120 minutos após o início do tratamento; momento 180 minutos (M180’), 180 minutos após o início do tratamento.

O exame clínico seguiu as recomendações de Feitosa ${ }^{11}$, com avaliação da frequência cardíaca (FC), frequência respiratória (FR). As colheitas de sangue foram realizadas por meio de venipunctura da jugular externa utilizando-se tubos heparinizados para análise hemogasométrica seguindo recomendações 
de Sucupira e Ortolani ${ }^{12}$. Imediatamente após a colheita de suco ruminal foi mensurado $\mathrm{pH}$ (phmetro marca selm ${ }^{\circledast}$ ). A determinação de ácido láctico total foi realizada segundo a técnica colorimétrica descrita por Pryce ${ }^{13}$.

As amostras de sangue total, provenientes dos tubos com EDTA, foram analisadas segundo técnica do micro-hematócrito para determinação do volume globular.

A determinação da osmolaridade sérica e ruminal foram obtidas em osmomêtro por ponto de congelamento (Advanced ${ }^{\mathrm{Tm}}$ Instruments, modelo The Advanced Micro-Osmometer 3300).

As análises estatísticas foram realizadas intragrupo, considerando o momento zero como controle dentro dos grupos tratado e controle; e entre grupos, comparando os dois grupos em cada um dos momentos avaliados. Todos os dados foram testados quanto à sua distribuição, utilizando-se o teste de Kolmogorov-Smirnov. Os dados que tiveram distribuição normal foram submetidos à análise de variância (teste F), com os grupos comparados pelo teste de Tukey ou T de Student ${ }^{14}$. Os dados com distribuição não-paramétrica foram avaliados pelo teste de Mann Whitney e expressos por mediana14. Foi adotado grau de significância mínimo de $5 \%$ e as análises estatísticas foram realizadas com auxílio de software estatístico ${ }^{15}$.

\section{Resultados e Discussão}

O modelo experimental conseguiu provocar uma acidose ruminal de grau médio a moderado, atingindo $\mathrm{pH}$ entre 4,2 a 4,8 e concentração de ácido láctico ruminal de 55 a $100(\mathrm{mM} / \mathrm{L})$. A acidose metabólica sistêmica ocorreu em todos os momentos mais críticos do experimento, do M20h ao M180', apenas de grau moderado, com média de pH 7,24 (Tabela 2), visto que a concentração de ácido láctico sanguíneo não atingiu valores muito altos, com médias de $10 \mathrm{mMol} / \mathrm{L}$.Estes resultados diferiram de outros estudos $^{16,17}$ seguindo o mesmo modelo experimental, principalmente devido as mudanças no manejo de adaptação que foram realizadas para atender sugestões emanadas pela Comissão de Bioética da FMVZ-USP, visando provocar uma acidose de grau mais brando nos animais.

A rápida infusão intravenosa de um grande volume de SSH não provocou quaisquer efeitos colaterais ou mal-estar nos animais. O número de batimentos cardíacos não foi alterado durante a aplicação de SSH (Tabela 1). Constable ${ }^{5}$ mencionou que concentrações de SSH muito elevadas podem causar quadro de bra-

Tabela 1 - Valores médios e desvio padrão da frequência cardíaca (FC) (bpm) e frequência respiratória $(\mathrm{FR})(\mathrm{mpm})$ dos bovinos dos grupos tratado e controle nos diferentes momentos - São Paulo- 2009

\begin{tabular}{|c|c|c|c|c|c|c|c|c|}
\hline \multirow[t]{3}{*}{ Momentos } & \multicolumn{4}{|c|}{ Grupo Tratamento (SSH) } & \multicolumn{4}{|c|}{ Grupo Controle (SSI) } \\
\hline & \multicolumn{2}{|c|}{$\mathrm{FC}$} & \multicolumn{2}{|c|}{ FR } & \multicolumn{2}{|c|}{$\mathrm{FC}$} & \multicolumn{2}{|c|}{ FR } \\
\hline & Média & DP & Média & DP & Média & $\mathrm{DP}$ & Média & $\mathrm{DP}$ \\
\hline M 0 & $66,0^{\mathrm{B}}$ & 20,8 & 20 & 4,4 & $68^{\text {B }}$ & 13,3 & 22 & 4,5 \\
\hline M 20h & $115,7^{\mathrm{A}}$ & 18,3 & 23 & 3,3 & $102,7^{\mathrm{A}}$ & 14,7 & 23 & 6,0 \\
\hline M 30' & $117,3^{\mathrm{A}}$ & 21,9 & 20 & 5,4 & $96,0^{\mathrm{A}}$ & 7,5 & 20 & 6,9 \\
\hline M 60' & $110,7^{\mathrm{A}}$ & 15,3 & 23 & 13,5 & $111,3^{\mathrm{A}}$ & 16,7 & 25 & 7,8 \\
\hline M 120' & $114,0^{\mathrm{A}}$ & 15,0 & 23 & 5,3 & $113,3^{\mathrm{A}}$ & 20,7 & 22 & 4,9 \\
\hline M 180' & $110,0^{\mathrm{A}}$ & 15,8 & 21 & 3,0 & $106,7^{\mathrm{A}}$ & 25,3 & 18 & 2,2 \\
\hline$P$ & \multicolumn{2}{|c|}{0,001} & \multicolumn{2}{|c|}{0,86} & \multicolumn{2}{|c|}{0,001} & \multicolumn{2}{|c|}{0,42} \\
\hline
\end{tabular}

Letras maiúsculas distintas nas colunas indicam diferenças significativas entre os momentos para cada variável. Não foram observadas diferenças entre grupos para as variáveis FC e FR 
Tabela 2 - Valores médios e desvio padrão do pH sanguíneo e da pressão parcial de gás carbônico $\left(\mathrm{pCO}_{2}-\mathrm{mmHg}\right)$ dos bovinos dos grupos tratado e controle nos diferentes momentos - São Paulo - 2009

\begin{tabular}{|c|c|c|c|c|c|c|c|c|}
\hline \multirow[t]{3}{*}{ Momentos } & \multicolumn{4}{|c|}{ Grupo Tratamento (SSH) } & \multicolumn{4}{|c|}{ Grupo Controle (SSI) } \\
\hline & \multicolumn{2}{|c|}{$\mathrm{ph}$} & \multicolumn{2}{|c|}{$\mathrm{PCO}_{2}$} & \multicolumn{2}{|c|}{$\mathrm{ph}$} & \multicolumn{2}{|c|}{$\mathrm{PCO}_{2}$} \\
\hline & Média & DP & Média & $\mathrm{DP}$ & Média & DP & Média & $\mathrm{DP}$ \\
\hline M 0 & $7,36^{\mathrm{A}}$ & 0,04 & $52,9^{\mathrm{A}}$ & 3,6 & $7,35^{\mathrm{A}}$ & 0,02 & $49,1^{\mathrm{A}}$ & 7,9 \\
\hline $\mathrm{M} 20 \mathrm{~h}$ & $7,24^{\text {в }}$ & 0,05 & $42,8^{\text {в }}$ & 5,5 & $7,24^{\mathrm{B}}$ & 0,04 & $42,1^{\mathrm{AB}}$ & 2,5 \\
\hline M 30' & $7,21^{\mathrm{B}}$ & 0,05 & $43,3^{\text {в }}$ & 4,4 & $7,24^{\mathrm{B}}$ & 0,04 & $41,4^{\mathrm{AB}}$ & 4,0 \\
\hline M 60' & $7,22^{\mathrm{B}}$ & 0,05 & $43,9^{\mathrm{B}}$ & 6,7 & $7,24^{\mathrm{B}}$ & 0,04 & $41,9^{\mathrm{AB}}$ & 4,9 \\
\hline M 120' & $7,24^{\mathrm{B}}$ & 0,05 & $42,5^{\text {в }}$ & 7,7 & $7,25^{\text {в }}$ & 0,05 & $39,5^{\mathrm{B}}$ & 3,4 \\
\hline M 180' & $7,26^{\mathrm{B}}$ & 0,05 & $38,5^{\mathrm{B}}$ & 6,4 & $7,26^{\mathrm{B}}$ & 0,05 & $37,9^{\mathrm{B}}$ & 3,2 \\
\hline$P$ & \multicolumn{2}{|c|}{0,001} & \multicolumn{2}{|c|}{0,004} & \multicolumn{2}{|c|}{0,001} & \multicolumn{2}{|c|}{0,004} \\
\hline
\end{tabular}

Letras maiúsculas distintas nas colunas indicam diferenças significativas entre os momentos para cada variável. Não foram observadas diferenças entre grupos para as variáveis ph e $\mathrm{pCO}_{2}$

dicardia, porém dentro das concentrações usuais, como a empregada no presente trabalho, tal efeito praticamente não é visto, mesmo quando a infusão é feita em alta velocidade. Foi observada elevação na freqüência cárdica quando do estabelecimento quadro de acidose láctica (M20h) e no decorrer dos momentos de tratamento, com um incremento de cerca de $75 \%$ em relação ao número de batimentos basal. Tal elevação de FC é descrita como sintoma de acidose láctica ${ }^{3,17}$. Não foi constatada nenhuma alteração na frequência respiratória no decorrer do experimento ou por influência do tratamento ( $\mathrm{Ta}$ bela 1). Geralmente, é descrita bradipneia em casos acidose láctica metabólica ${ }^{17}$, em especial quando os valores de $\mathrm{pH}$ sanguíneo são inferiores a 7,18 ${ }^{1}$. Em nenhum momento os garrotes do experimento apresentaram acidose metabólica de tal magnitude. Segundo Constable ${ }^{5}$ a infusão de SSH mesmo em alta velocidade, como foi a utilizada neste presente experimento, não alterou a frequência respiratória.

É notório também frisar que, embora ruminantes com acidose láctica ruminal aguda não desenvolvam uma hipocapnia compensatória para aliviar a acidose sistêmica, a SSH gerou uma discreta e ligeira hipercapnia durante o tratamento, dissipando-se em seguida (Tabela 2). Essa hipercapnia também foi descrita em porcos anestesiados com isofluorano e submetidos a choque hemorrágico, sendo em seguida tratados com $\mathrm{SSH}^{18}$. Esses autores atribuíram a hipercapnia à queda na frequência respiratória e consequente hipoventilação após o tratamento com SSH. No presente trabalho, existiu realmente uma numérica, mas não significativa, diminuição na frequência respiratória após a infusão com SSH. Mas o mesmo fenômeno ocorreu e com idêntica magnitude nos animais tratados com SSI. Provavelmente, essa reação observada por Moon e Kramer ${ }^{18}$ tenha forte influência da interação anestesia e tratamento com SSH, visto que em animais não anestesiados tal efeito não foi visto. A anestesia per se já tem uma ação bradipneica e é possível que nesta condição específica a SSH amplie este processo. Assim, especula-se que a hipercapnia em animais tratados com SSH possa ter outra gênese.

Uma possível hipótese se para a hipercapnia é devido excesso de cloro, Block ${ }^{19}$ alimentou vacas no final da gestação com dietas francamente aniônica, ricas em cloreto e constatou além da hipercloremia uma discreta hipercapnia e acidemia. Esse autor atribuiu a gênese desse status ao processo denominado chloride shift, em que o excesso de cloreto no meio pulmonar, por um complexo mecanismo, inibe levemente a excreção de $\mathrm{CO}_{2}$ pelo ar expirado. 
Tabela 3 - Valores médios e desvio padrão da osmolaridade ruminal (OR) mOsm/L e osmolaridade sérica (OS) $\mathrm{mOsm} / \mathrm{L}$ dos bovinos dos grupos tratado e controle nos diferentes momentos - São Paulo - 2009

\begin{tabular}{|c|c|c|c|c|c|c|c|c|}
\hline \multirow[t]{3}{*}{ Momentos } & \multicolumn{4}{|c|}{ Grupo Tratamento (SSH) } & \multicolumn{4}{|c|}{ Grupo Controle (SSI) } \\
\hline & \multicolumn{2}{|c|}{ OR } & \multicolumn{2}{|c|}{ OS } & \multicolumn{2}{|c|}{ OR } & \multicolumn{2}{|c|}{ Os } \\
\hline & Média & DP & Média & DP & Média & DP & Média & DP \\
\hline M 0 & $275^{\mathrm{BC}}$ & 27 & 309 & 11 & $259^{\mathrm{B}}$ & 29 & 300 & 5 \\
\hline M $20 \mathrm{~h}$ & $343^{\mathrm{A}}$ & 12 & 308 & 17 & $336^{\mathrm{A}}$ & 29 & 304 & 6 \\
\hline M 30' & $258^{\mathrm{C}}$ & 16 & $321 \mathrm{a}$ & 13 & $275^{\text {в }}$ & 19 & $309 b$ & 10 \\
\hline M 60' & $297^{\text {в }}$ & 23 & $318 \mathrm{a}$ & 12 & $292^{\text {в }}$ & 21 & $301 b$ & 6 \\
\hline M 120' & $300^{\mathrm{B}}$ & 10 & 307 & 21 & $296^{\mathrm{B}}$ & 16 & 298 & 22 \\
\hline M 180' & $308^{\text {в }}$ & 9 & 307 & 12 & $297^{\text {B }}$ & 15 & 300 & 7 \\
\hline
\end{tabular}

A infusão de $\mathrm{SSH}$ provocou nos animais com acidose alterações no movimento de fluidos no organismo. Existiram evidências de que a água administrada no rúmen foi de alguma forma mais absorvida para a circulação que nos medicados com SSI. Como era esperada, a administração de água no rúmen gerou, 30 minutos após a sua infusão, uma significativa queda na osmolaridade ruminal em ambos os tratamentos (Tabela 3). Examinando-se essa tabela, nota-se que não existiu diferença significativa entre os tratamentos nos momentos subsequentes, ou seja, no M60', M120' e M180'. Po-

Tabela 4 - Valores médios e desvio padrão do volume globular (VG) (\%) dos bovinos dos grupos tratado e controle nos diferentes momentos - São Paulo - 2009

\begin{tabular}{|c|c|c|c|c|}
\hline \multirow[t]{3}{*}{ Momentos } & \multicolumn{4}{|c|}{ Volume Globular (\%) } \\
\hline & \multicolumn{2}{|c|}{$\begin{array}{c}\text { Grupo Tratamento } \\
\text { (SSH) }\end{array}$} & \multicolumn{2}{|c|}{$\begin{array}{c}\text { Grupo Controle } \\
\text { (SSI) }\end{array}$} \\
\hline & Média & $\mathrm{DP}$ & Média & $\mathrm{DP}$ \\
\hline M 0 & $28,6 \mathrm{~B}$ & 1,9 & 28,8 & 1,7 \\
\hline M $20 \mathrm{~h}$ & $36,0 \mathrm{~A}$ & 3,9 & 33,3 & 4,8 \\
\hline M 30' & $28,2 \mathrm{~B}$ & 2,8 & 30,2 & 3,9 \\
\hline M 60' & $28,8 \mathrm{~B}$ & 2,7 & 30,2 & 3,8 \\
\hline M 120' & $29,0 \mathrm{~B}$ & 2,2 & 29,5 & 3,3 \\
\hline M 180' & $28,5 \mathrm{~B}$ & 2,4 & 28,3 & 4,0 \\
\hline$P$ & \multicolumn{2}{|c|}{0,001} & \multicolumn{2}{|c|}{0,358} \\
\hline
\end{tabular}

Letras maiúsculas distintas nas colunas indicam diferenças significativas entre os momentos para cada variável. Não foram observadas diferenças entre grupos para a variável VG rém, acompanhando a tabela 5 , que indica a diferença de osmolaridade entre o M30' e os momentos subsequentes, destaca-se que tanto no M60, M120' e M180' esta diferença foi superior nos animais medicados com SSH. O volume globular não obteve diferença significativa entre os tratamentos, pois foi comparado em relação ao momento basal MO (Tabela 4). Objetivando uma melhor comparação entre os grupos SSI e SSH, visando retirar a interferência da variação individual no M20, foi realizado cálculo da diferença do VG entre os momentos de tratamento (M30; M60; M120; M180) em relação ao pico do quadro de acidose (M20) (Tabela 5). Concomitantemente, a osmolaridade, a diferença entre o volume globular do M20h e os momentos subsequentes (M30' até M120') também foi superior nos bovinos infundidos com SSH (Tabela 5).

Essas significativas diferenças de osmolaridade ruminal e de volume globular, em pequeno espaço de tempo, indicam que ocorreu uma maior absorção de fluidos do rúmen, com posterior passagem para o espaço vascular. Tal fenômeno deve ter ocorrido, pois logo após a infusão intravascular de SSH existiu uma maior osmolaridade no sangue, em relação aos medicados com SSI. Analisando as medianas entre a 
Tabela 5 - Valores médios e desvio padrão da diferença dos valores da osmolaridade ruminal (OR) (mOsm/L) entre os momentos de tratamento (M60'; M120'; M180') em relação ao M30' e diferença do volume globular VG (\%) entre os momentos de tratamento (M30' até M180') em relação ao M20h - São Paulo - 2009

\begin{tabular}{|c|c|c|c|c|c|c|c|c|}
\hline \multirow[t]{3}{*}{ Momentos } & \multicolumn{4}{|c|}{ Grupo Tratamento (SSH) } & \multicolumn{4}{|c|}{ Grupo Controle (SSI) } \\
\hline & \multicolumn{2}{|c|}{ OR } & \multicolumn{2}{|c|}{ VG } & \multicolumn{2}{|c|}{ OR } & \multicolumn{2}{|c|}{ VG } \\
\hline & Média & $\mathrm{DP}$ & Média & $\mathrm{DP}$ & Média & $\mathrm{DP}$ & Média & $\mathrm{DP}$ \\
\hline M 30' & & & $7,83^{\text {a }}$ & 1,17 & & & $3,17^{\mathrm{b}}$ & 1,72 \\
\hline M 60' & $39^{\mathrm{a}}$ & 18 & $7,17^{\text {a }}$ & 1,60 & $17^{\mathrm{b}}$ & 5 & $3,17^{\mathrm{b}}$ & 1,94 \\
\hline M120' & $42^{\mathrm{a}}$ & 12 & $7,00^{\text {a }}$ & 2,19 & $21^{b}$ & 6 & $3,83^{b}$ & 2,71 \\
\hline M 180’ & $49^{\mathrm{a}}$ & 16 & 7,50 & 3,15 & $23^{\mathrm{b}}$ & 5 & 5,00 & 2,53 \\
\hline
\end{tabular}

diferença entre OR e a OS, obteve-se diferença significativa $(P=0,005)$ apenas no $M 30$, Esse aumento de osmolaridade sanguínea coincidiu com uma queda na osmolaridade ruminal gerando um superávit sanguíneo, que favoreceu a passagem de fluidos para seu espaço. Conforme Constable ${ }^{5}$ relata, a SSH tem uma ação efêmera.

Esses resultados indicam que a infusão de solução hipertônica associada com administração de água no rúmen favorece a transferência de fluidos do rúmen para a circulação, contribuindo para a correção do estado de desidratação. Provavelmente, os efeitos benéficos da infusão de solução salina saturada sejam mais evidentes em bovinos com acidose que apresentem quadro de desidratação mais pronunciado. Tal protocolo medicamentoso necessita ser testado em situações mais desafiadoras.

\section{Referências}

1. MARUTA, C. A.; ORTOLANI, E. L. Susceptibilidade de bovinos das raças Jersey e Gir à acidose láctica ruminal: II acidose metabólica e metabolização do lactato-L. Ciência Rural, v. 32, n. 1, p. 61-65, 2002.

2. MENDES NETTO, D.; ORTOLANI, E. L. Blood acidbase status evaluation of sodium bicarbonate and lactated Ringer's solution for the treatment of ruminal lactic acidosis. Veterinária Notícias, v. 6, n. 2, p. 31-39, 2000.

3. RADOSTITS, O. M.; GAY, C. C.; HINCHCLIFF, K. W.; CONSTABLE, P. D. Veterinary medicine. 10. ed. Edimburgo: Saunders Elsevier, 2007. 2156 p.

\section{Conclusão}

O tratamento não provocou quaisquer efeitos colaterais. Ocorreu temporariamente uma discreta acidemia, acompanhada de leve hipercapnia, sem agravamento no quadro de acidose sistêmica. Podendo a solução salina hipertônica a 7,5\% ser usada em quadros de acidose láctica ruminal aguda sem agravamento da já instalada acidose sistêmica. Em casos de ALRA, pode ser utilizada como tratamento adicional para melhora no quadro de desidratação, que é relativamente frequente nesta enfermidade.

\section{Agradecimentos}

Frederico A. M. L. Rodrigues, Antonio H. H. Minervino e Leonardo Frasson dos Reis são gratos à FAPESP pelas bolsas de pós-graduação concedidas e Auxílio Pesquisa.

4.VELASCO, I. T.; PONTIERI, V.; ROCHA E SILVA JR., M.; LOPES, O. U. Hyperosmotic $\mathrm{NaCl}$ and severe hemorrhagic shock. American Journal of Physiology, v. 239, n. 5, p. 664673, 1980.

5. CONSTABLE, P. D. Hypertonic saline. Veterinary Clinics of North America: Food Animal Practice, v. 15, n. 3, p. 559-585, 1999.

6. TYLER, J. W.; WELLES, E. G.; ERSKINE, R. J.; LIN, H. C.; WILLIAN, M. A.; SPANO, J. S.; GASLIN, J. T.; MCCLURE, K. A. Clinical and clinicopathologic changes in cows with endotoxin-induced mastitis treated with small volumes 
of isotonic or hypertonic sodium chloride administred intravenously. American Journal of Veterinary Research, v. 55 , n. 2, p. 278-287, 1994.

7. BENESI, F. J.; HOWARD, D. L.; SANCHEZ, F. B.; CARDOSO DE SÁ, C. S.; TEIXEIRA, C. M.; LEAL, M. L. R.; LISBOA, J. A. N.; MIRANDOLA, R. M. S. Tratamento da endotoxemia experimental em bezerros. Estudo de alternativas terapêuticas. A Hora Veterinária, v. 22, n. 2. p. 81-88, 2000.

8. ROEDER, B. L.; SU, C.; SCHAALJE, B. Acute effects of intravenously administered hypertonic saline solution on transruminal rehydration in dairy cows. American Journal of Veterinary Research, v. 58, n. 5, p. 549-559, 1997.

9. HUBER, T. L. Effect of acute indigestion on compartmental water volumes and osmolality in sheep. American Journal Veterinary Research, v. 32, n. 6, p. 887-890, 1971.

10. ORTOLANI, E. L. Induction of lactic acidosis in cattle with sucrose: Relationship between dose, rumen fluid $\mathrm{pH}$ and animal size. Veterinary and Human Toxicology, v. 37, n. 5, p. 462-464, 1995.

11.FEITOSA, F. L. F. Semiologia Veterinária: a arte do diagnóstico. São Paulo: Rocca, 2004. 824 p.

12.SUCUPIRA, M. C. A.; ORTOLANI, E. L. Uso de sangue arterial e venoso no exame do equilíbrio ácido básico de novilhos normais ou com acidose metabólica. Ciência Rural, v. 33 , n. 5 , p. $863-868,2003$.
13.PRYCE, J. D. A modification of the Barker-Summerson method for the determination of lactic acid. Analyst, v. 94, n. 125 , p. 1151-1152, 1969.

14.SAMPAIO, I. B. M. Estatística aplicada à experimentação animal. 2. ed. Belo Horizonte: Fundação de Estudo e Pesquisa em Medicina Veterinária e Zootecnia, 2002. 265 p.

15. MINITAB. The student edition of MINITAB Statistical software adapted for education. Release 13.0. User's Manual. New York: Addison-Wesley, 2000. CD-ROM.

16.LEAL, M. R. L; MARUTA, C. A.; ORTOLANI, E. L. Uso de bicarbonato e lactato-L para correção da acidose metabólica sistêmica em bovinos com acidose láctica ruminal aguda. Arquivo Brasileiro de Medicina Veterinária e Zootecnia, v. 59, n. 4, p. 971-976, 2007.

17. ORTOLANI, E. L.; MARUTA, C. A.; MINERVINO, A. H. H. Aspectos clínicos da indução experimental de acidose láctica ruminal em zebuínos e taurinos. Brazilian Jounal of Veterinary Research and Animal Science, v. 47, n. 4, p. 253-261, 2010.

18.MOON, P. F.; KRAMER, G. C. Hypertonic saline-dextran resuscitation from hemorrhagic shock induces transient mixed acidosis. Critical Care Medicine, v. 23, n. 2, p. 323-331, 1995.

19. BLOCK, E. Manipulation of dietary cation-anion difference on nutritionally rated production diseases, productivity, and metabolic responses of dairy cows. Journal of Dairy Science, v. 77, n. 5 , p. $1437-1450,1994$. 